\title{
Reproductive Endocrinology
}

\author{
Lena Sahlin and Olle Söder \\ Paediatric Endocrinology Unit, Department of Women's and Children's Health, Astrid Lindgren Children's \\ Hospital, Karolinska Institutet and Karolinska University Hospital, Stockholm, Sweden
}

\begin{abstract}
Reproductive endocrinology is a wide and lively research field attracting considerable attention because of its implications in everyday life. More than 1,000 articles published in the past year were found under the search words used, of which 22 papers were selected. This chapter hosts a variety of papers dealing with many important aspects of reproductive endocrinology including the function of new and old genes, puberty, gonads, stem cells for gametogenesis, impact of lifestyle and environmental factors on reproduction, placental origin of psychiatric disorders, reproductive behavior and others. Some other chapters of this book may also host publications dealing with subjects related to reproduction. There are of course many other excellent articles published within the field of reproductive endocrinology during the past year, some that we might have missed in our search and yet others which were not possible to include due to space limitation. The aim has been to present a mix of experimental and clinical publications advancing our knowledge within the field of reproductive endocrinology. The selected papers obviously represent our own bias but we hope you find some of them interesting to read and helpful for your daily work in clinical and experimental paediatric endocrinology.
\end{abstract}

\section{New mechanisms - and their later outcome}

\section{Central precocious puberty caused by mutations in the imprinted gene MKRN3}

Abreu AP, Dauber A, Macedo DB, Noel SD, Brito VN, Gill JC, Cukier P, Thompson IR, Navarro VM, Gagliardi PC, Rodrigues T, Kochi C, Longui CA, Beckers D, de Zegher F, Montenegro LR, Mendonca BB, Carroll RS, Hirschhorn JN, Latronico AC, Kaiser UB

Division of Endocrinology, Diabetes, and Hypertension, Brigham and Women's Hospital and Harvard Medical School, Boston, MA, USA

N Engl J Med 2013;368:2467-2475

Background: The onset of puberty is first detected as an increase of the pulsatile secretion of gonadotropin-releasing hormone $(\mathrm{GnRH})$. Too early activation of the hypothalamic-pituitary-gonadal axis results in central precocious puberty (CPP). The timing of pubertal development is driven in part by genetic factors, but only a few, rare molecular defects associated with CPP have been identified.

Methods: A whole-exome sequencing in 40 members of 15 families with CPP was executed. Candidate variants were confirmed with Sanger sequencing. Quantitative real-time polymerase-chain-reaction assays to determine levels of messenger RNA (mRNA), in the hypothalami of mice at different ages, were also performed.

Results: Four novel heterozygous mutations in MKRN3 were found in 5 of the 15 families; both sexes were affected. MKRN3 is the gene encoding makorin RING-finger protein 3 . The mutations included three frameshift mutations, predicted to encode truncated proteins, and one missense mutation, predicted to disrupt protein function. MKRN3 is a paternally expressed, imprinted gene located in the Prader-Willi syndrome critical region. All affected persons inherited the mutations from their fathers, a finding that indicates perfect segregation with the mode of inheritance expected for this imprinted gene. Levels of Mkrn3 mRNA were high in the arcuate nucleus of prepubertal mice, decreased immediately before puberty, and remained low after puberty.

Conclusion: Deficiency of MKRN3 causes CPP in humans. 


\section{Association of aromatase (TTTA) $n$ repeat polymorphisms with central precocious puberty in girls}

Lee HS, Kim KH, Hwang JS

Department of Pediatrics, Ajou University School of Medicine, Ajou University Hospital, Suwon, Korea

Clin Endocrinol (Oxf) 2014; DOI: 10.1111/cen.12439

Background: Central precocious puberty (CPP) is characterized by early activation of the pituitary-gonadal axis. Estrogen is the final key factor to start the onset of puberty in girls. The cytochrome P450 19A1 (CYP19A1) gene encodes an aromatase that is responsible for the conversion of androgens to estrogen, which is a key step in estrogen biosynthesis. The aim of this study was to identify CYP19A1 gene mutations or polymorphisms in girls with CPP.

Methods: The frequency of allelic variants of the CYP19A1 exons and the tetranucleotide tandem repeat (TTTA) $n$ was evaluated in intron 4 in 203 idiopathic CPP girls and 101 normal healthy women.

Results: The genotype analysis of the CYP19A1 (TTTA) $)_{n}$ polymorphism revealed six different alleles ranging from 7 to 13 repeats. Among the 6 different repeat alleles detected in this study, the (TTTA)13 repeat allele was only detected in the patient group and the carriers of the (TTTA)13 allele were significantly associated with an increased risk of CPP. Carriers of the (TTTA)13 repeat allele were significantly younger at pubertal onset and had higher levels of estrogen than non-carriers of the allele. Although nine polymorphisms were detected in exons of the CYP19A1 gene, no clinical significance was observed.

Conclusion: The carriers of a higher repeat (TTTA)13 polymorphism in intron 4 of the CYP19A1 gene had higher levels of estrogen and may thus have a higher risk of developing CPP.

In the two above studies, genetic analyses were performed in 40 members from 15 families with CPP, and in 203 girls with idiopathic CPP and 101 healthy women, respectively. The results from the first study show four novel heterozygous mutations in the MKRN3 gene, and that both sexes are affected. Thus, MKRN3 deficiency causes CPP in humans. In the second study it was found that girls harboring the (TTTA) 13 allele have a significantly higher risk of developing CPP, are younger at the onset of puberty and have higher levels of estrogen than individuals without this allele. The study by Lee et al. was commented on in Reproductive Endocrinology [1]. Hence, there are different mutations within the same gene, as well as different genes, that can cause CPP in both girls and boys. These studies expand our knowledge on the genetic background of CPP and add new genes to be investigated for the pediatric endocrinologist caring for CPP patients of familiar origin.

\section{Delayed puberty and estrogen resistance in a woman with estrogen receptor- $\alpha$ variant}

Quaynor SD, Stradtman EW Jr, Kim HG, Shen Y, Chorich LP, Schreihofer DA, Layman LC

Section of Reproductive Endocrinology, Infertility, and Genetics, Department of Obstetrics and Gynecology, Institute of Molecular Medicine and Genetics, and the Neuroscience Program, Medical College of Georgia, Georgia Regents University, Augusta, GA, USA

N Engl J Med 2013;369:164-171

Background: Although androgen resistance has been characterized in men with a normal chromosome complement and mutations in the androgen-receptor gene, a mutation in the gene encoding estrogen receptor- $\alpha$ (ESR1) was previously described only in one man and not, to our knowledge, in any woman. Methods: A case description of an 18-year-old woman without breast development and with markedly elevated serum levels of estrogens and bilateral multicystic ovaries is presented. Serum estradiol was measured by immunoassay, but estradiol and estrone were also measured by isotope dilution high-performance liquid chromatography-mass spectrometry (LC-MS). DNA from peripheral blood white cells was sequenced with the use of polymerase chain reaction (PCR) assay for protein-coding exons in ESR1 (GenBank accession No. NM_000125), followed by analysis by Basic Local Alignment Search Tool (BLAST) software and Clustal alignment. The putative mutation was analyzed in 192 DNA samples obtained from white controls.

Results: DNA sequencing of ESR1 revealed a homozygous mutation, c.1125G $\rightarrow \mathrm{T}$ in exon 5, corresponding to p.Gln375His, which was absent in the single-nucleotide polymorphism database (dbSNP) and in 
192 samples provided by white controls. This loss-of-function ESR1 mutation was located in a completely conserved residue and was found to interfere with estrogen signaling. The clinical presentation was similar to that of the mouse orthologue knockout.

Conclusion: This case shows that disruption of ESR1 causes profound estrogen resistance in women.

This study, also highlighted in Nature Reviews in Endocrinology [2], presents a case of severe estrogen resistance caused by an ESR1 mutation. It was generally assumed that lack of a functioning estrogen receptor (only $\alpha$ known at the time) was incompatible with life [3], but 20 years ago the first report of a man with estrogen resistance was published, showing that the cause was an ESR1 mutation [4]. The present study is the first report of an ESR1 mutation in a woman, and it shows once again that ESR1 mutations are not lethal but could result in a profoundly estrogen-resistant state. The prevalence of ESR1 mutations in humans is unknown but are probably rare, the few cases found so far support such statement. If there are milder ESR1 mutations that could cause an incomplete estrogen-deficient state remains to be determined. In this particular case the presence of bi-allelic mutations suggests that the patient's estrogen resistance is autosomal recessive, especially since microarray analysis showed a region consistent with a parental history of a second-degree relation. The study gives reasons to test for ESR1 mutations in girls presenting an absent or severely delayed breast development and puberty, despite high estrogen levels. Further longitudinal phenotyping of this index case is of course of great interest.

\title{
Treated and untreated women with idiopathic precocious puberty: long-term follow-up and reproductive outcome between the third and fifth decades
}

\author{
Lazar L, Meyerovitch J, de Vries L, Phillip M, Lebenthal Y \\ The Jesse $Z$ and Sara Lea Shafer Institute for Endocrinology and Diabetes, National Center for Childhood Diabetes, \\ Schneider Children's Medical Center of Israel, Petah Tikva, and Sackler Faculty of Medicine, Tel Aviv University, \\ Tel Aviv, Israel \\ Clin Endocrinol (Oxf) 2014;80:570-576
}

Background: Central precocious puberty (CPP), treated or untreated, may have implications in adulthood. This study was performed to assess the reproductive outcome and social adjustment after CPP.

Methods: This was a cross-sectional study of former CPP women between the 3rd and 5th decades of life. Demographic data and gynaecological history of 214 CPP women aged 25-56 years and of 446 controls with normal puberty, matched for age and year of birth, were recorded in a structured interview. Of the CPP women, 135 were $\mathrm{GnRH}$ analogue ( $\mathrm{GnRHa}$ )-treated, 18 cyproterone acetate $(\mathrm{CyA})$-treated, and 61 untreated.

Results: Marital status, education and number of children were similar in CPP women and in controls. Clinical hyperandrogenism (acne/hirsutism with oligomenorrhoea) was more frequently reported in CPP women than in controls, and there were significant differences between each CPP treatment group compared to controls: GnRH analogue-treated 29.6 vs. $17.4 \%$, cyproterone acetate-treated 50 vs. $20.4 \%$, untreated 34.4 vs. $17.2 \%$, but with no significant difference between the CPP groups. Spontaneous pregnancy was similarly achieved by treated CPP and controls: GnRHa-treated 90.4 vs. 93.4\%, CyA-treated 86.7 vs. $90.2 \%$. Assisted fertilization rate was significantly higher in untreated CPP than in treated CPP groups and controls. Untreated CPP was the only group associated with both clinical hyperandrogenism and fertility problems. Pregnancy was uneventful in $90.2 \%$ of CPP women and $90.9 \%$ of controls.

Conclusion: The increased rate of clinical hyperandrogenism among CPP women implies that the underlying neuroendocrine dysfunction persists into adult life. Pubertal suppression treatment may have some protective effect as fertility problems were more prevalent only among untreated CPP women. Educational achievements and marital status were unaffected by CPP.

In this paper the authors have studied the long-term reproductive and social outcome of adult women aged 25-56 years with a history of CPP. There seems to be no major differences between girls treated with $\mathrm{GnRH}$ analogues or cyproterone acetate as compared to controls, whereas the untreated CPP girls experienced both a higher prevalence of hyperandrogenism and menstrual irregularity. The untreated CPP girls had significantly fewer spontaneous pregnancies as compared to controls (67 vs. 
$87 \%$ ). The take-home message is that treatment of young girls with CPP seems to be beneficial for most parameters studied, and that there may be an underlying neuroendocrine dysfunction, at least in some girls, that persists into adult life. Although the reproductive outcome in early and midadulthood was found to be normal in the great majority of the studied patients, continued follow-up of former CPP women is needed to determine whether CPP has even longer-term health implications, for example on the risk of premature ovarian failure and premature menopause in the late reproductive years.

\section{New hope}

\section{Reproductive stem cell differentiation: extracellular matrix, tissue microenvironment, and growth factors direct the mesenchymal stem cell lineage commitment}

Vidane AS, Zomer HD, Oliveira BM, Guimaraes CF, Fernandes CB, Perecin F, Silva LA, Miglino MA, Meirelles FV, Ambrosio CE

Sector of Animal Anatomy, Department of Surgery, Faculty of Veterinary Medicine and Animal Sciences, University of Sao Paulo, Sao Paulo, Brazil

Reprod Sci 2013;20:1137-1143

Background: Mesenchymal stem cells (MSCs) are of interest in regenerative medicine due to their high capability to proliferate and differentiate in multiple specialized lineages under defined conditions. The reproductive system is considered an important source of MSCs. Many factors have been reported as critical for cell lineage specification and determination but this knowledge need to be expanded to increase the clinical usefulness of this technology.

Methods: This review discusses the biology and potential usefulness of MSCs with relation to the reproductive system. The effects of extracellular matrix, tissue environment and growth factors for cell lineage commitment are discussed, with relevance for reproductive lineage stem cells.

Results: MSCs responses to various stimuli from culture medium and soluble factors probably occur through several intracellular activation pathways. The molecular mechanisms in which the cells respond to these mechanical or chemical influences still remain elusive. Recent findings suggest a synergistic effect of the microenvironment and soluble cell culture factors affecting cell differentiation and lineage specification. This review gives an updated overview of important concepts in this area of research.

Conclusion: For future clinical applications in cell therapy and regenerative medicine, detailed protocols of reproductive MSCs differentiation need to be established.

Mesenchymal stem cells (MSCs) are pluripotent adult somatic stem cells derived from bone marrow and a wide variety of organs, including in the reproductive system, such as endometrium, ovary and placenta. MSCs are becoming of increasing clinical interest because of their potential in regenerative medicine. Collection and cryopreservation of MCSs from the placenta at birth has become a clinical (and commercial) application of this increased knowledge, despite that their clinical usefulness still awaits further developments. Principles of reproductive cell lineage specification of MSCs are described and discussed in some detail. This paper gives an overview of the state of the science of MSCs in relation to the reproductive field. This knowledge is important for the paediatric endocrinologist who wants to be updated on this expanding field. 


\section{A tripartite transcription factor network regulates primordial germ cell specification in mice}

Magnusdottir E, Dietmann S, Murakami K, Gunesdogan U, Tang F, Bao S, Diamanti E, Lao K, Gottgens B, Azim Surani M

Wellcome Trust/Cancer Research UK Gurdon Institute, University of Cambridge, Cambridge, UK Nat Cell Biol 2013;15:905-915

Background: Primordial germ cells (PGCs) are gonocyte precursors populating the primitive indifferent gonadal anlagen in early embryogenesis. A fundamental property of early germ cells is the unique epigenetic changes that ensue following PGC specification, leading to global erasure of DNA methylation and acquisition of a basal epigenetic state. Transitions in cell states are controlled by combinatorial actions of transcription factors. BLIMP1, the key regulator of PGC specification, apparently acts together with PRDM14 and AP2 $\gamma$. The authors aimed to investigate their individual and combinatorial functions.

Methods: The investigators set up an in vitro system for transcriptional readouts and chromatin immunoprecipitation sequencing analysis. They then integrated this data with information from single-cell transcriptome analysis of normal and mutant PGCs.

Results: The results show that BLIMP1 binds directly to repress somatic and cell proliferation genes. It also directly induces AP2 $\gamma$, which together with PRDM14 initiates the PGC-specific fate. The occupancy of critical genes by AP $2 \gamma$, which when computed altogether both individually and cooperatively with those of BLIMP1 and PRDM14, was determined and revealed a tripartite mutually interdependent transcriptional network for PGCs.

Conclusion: BLIMP1, AP2 $\gamma$ and PRDM14 are sufficient for PGC specification and resetting of the epigenome towards a basal state.

This elegant mouse study detailed how only three transcription factors cooperate in the early embryo to drive the development of primordial germ cells (PGCs). The three transcription factors necessary for starting the PGC gene program were BLIMP1, PRDM14, and AP2 $\gamma$. The investigators also examined the regulation of various factors involved in global DNA demethylation, a key event following PGC specification, and showed how these same three transcription factors cooperate to create the regulatory milieu that leads to this erasure. The mechanisms that regulate this unique resetting of the epigenome in early germ cells could in principle be extended towards approaches for modifying epigenetic states of normal and diseased tissues, which may have important clinical implications for the future. This study was highlighted in Biology of Reproduction [5].

\section{Concepts revised}

\section{PRC1 coordinates timing of sexual differentiation of female primordial germ cells}

Yokobayashi S, Liang CY, Kohler H, Nestorov P, Liu Z, Vidal M, van Lohuizen M, Roloff TC, Peters AH

Friedrich Miescher Institute for Biomedical Research, Basel, Switzerland

Nature 2013;495:236-240

Background: In mammals, sex differentiation of primordial germ cells (PGCs) is determined by extrinsic cues from the environment. In mouse female PGCs, expression of lineage-specific markers is stimulated by retinoic acid gene 8 (Stra8) and meiosis is induced in response to retinoic acid provided from the mesonephros. Given the widespread role of retinoic acid signalling during development, the molecular mechanisms that enable PGCs to express Stra8 and enter meiosis in a temporally correct fashion are unknown. 
Methods and Results: The authors identified gene-dosage-dependent roles in PGC development for Ring1 and Rnf2, two central components of the Polycomb repressive complex 1 (PRC1). Both paralogues are essential for PGC development between days 10.5 and 11.5 of gestation in the mouse. Rnf2 is subsequently required in female PGCs to maintain high levels of Oct4 and Nanog expression, and to prevent premature induction of meiotic gene expression and entry into meiotic prophase. Chemical inhibition of retinoic acid signalling partially suppresses precocious Oct4 downregulation and Stra8 activation in Rnf2-deficient female PGCs. Chromatin immunoprecipitation analyses show that Stra8 is a direct target of PRC1 and PRC2 in PGCs.

Conclusion: These data demonstrate the importance of PRC1 gene dosage in PGC development and in coordinating the timing of sex differentiation of female PGCs by antagonizing extrinsic retinoic acid signalling.

The exact control of the timing of cellular events in embryogenesis is crucial for proper development. This study shows how gene dosage of two repressor genes in combination with hormonal (retinoic acid) paracrine stimulation from the local tissue environment control primordial germ cell (PGC) development and prevent their premature entry into meiosis. The molecular mechanisms described here are surprisingly simple and give hope that they can be controlled by molecular tools that can be tailored into drug candidates. As PGCs have also been proposed as stem cells for germ cell cancer such developments may have direct clinical implications for male infertility and testicular cancer.

\section{Review}

\section{Building an ovary: insights into establishment of somatic cell lineages in the mouse}

Nicol B, Yao HH

Laboratory of Reproductive and Developmental Toxicology, National Institute of Environmental Health Sciences,

Research Triangle Park, NC, USA

Sex Dev 2014 (Epub ahead of print) DOI:10.1159/000358072

Background: The molecular pathways that drive the differentiation of somatic cell populations in the gonads have been the subjects of intensive research over the past decade. It is now clear that ovarian differentiation is an active coordinated event driven by secreted factors including R-spondin1, WNT4, and follistatin, and transcriptional regulators such as $\beta$-catenin and FOXL2. These factors direct bipotential somatic cell lineages toward an ovarian fate and simultaneously suppress the emergence of testisdetermining signals.

Methods and Results: This review summarizes the molecular pathways responsible for establishment of the ovary and discusses the current hypotheses on the origin(s) of somatic cell lineages and how these somatic cells acquire the characteristics necessary for their function during ovarian development and maintenance.

Conclusion: This review is recommended for readers who want a comprehensive update on the cellular and molecular development of the ovary.

The previous hypothesis that ovarian development is a passive event following a predestined path without need for guidance by specific ovarian determining genes has now been abandoned. During the past decade a number of novel observations have made clear that the ovary develops actively by suppression of intragonadal events aiming to force the supporting somatic cell lineage (Sertoli, granulosa) to a testicular path. These surprising findings have helped to develop novel concepts for the control of peri- and postnatal tissue differentiation. This comprehensive review is recommended to readers who want a state of the science update on these novel concepts. 


\section{The ESHRE-ESGE consensus on the classification of female genital tract congenital anomalies}

Grimbizis GF, Gordts S, Di Spiezio Sardo A, Brucker S, De Angelis C, Gergolet M, Li TC, Tanos V, Brolmann H, Gianaroli L, Campo R

Congenital Uterine Anomalies (CONUTA) common ESHRE-ESGE Working Group, ESGE Central Office, Leuven, Belgium

Gynecol Surg 2013;10:199-212 \& Hum Reprod 2013;28:2032-2044

Background: Congenital malformations of the female genital tract are common miscellaneous deviations from normal anatomy with health and reproductive consequences. Until now, three systems have been proposed for their categorization, but all of them are associated with serious limitations. The European Society of Human Reproduction and Embryology (ESHRE) and the European Society for Gynaecological Endoscopy (ESGE) have established a common Working Group, under the name CONUTA (CONgenital UTerine Anomalies), with the goal of developing a new updated classification system.

Methods: A scientific committee was appointed to run the project, looking also for consensus among the scientists working in the field. The new system is designed and developed based on: (1) scientific research through critical review of current proposals and preparation of an initial proposal for discussion between the experts, $(2)$ consensus measurement among the experts through the use of the DELPHI procedure, and (3) consensus development by the scientific committee, taking into account the results of the DELPHI procedure and the comments of the experts.

Results: Almost 90 participants took part in the process of development of the ESHRE/ESGE classification system, contributing with their structured answers and comments. The ESHRE/ESGE classification system is based on anatomy. Anomalies are classified into the following main classes, expressing uterine anatomical deviations deriving from the same embryological origin: U0, normal uterus; U1, dysmorphic uterus; U2, septate uterus; U3, bicorporeal uterus; U4, hemi-uterus; U5, aplastic uterus; U6, for still unclassified cases. The main classes have been divided into subclasses expressing anatomical varieties with clinical significance. Cervical and vaginal anomalies are classified independently into subclasses having clinical significance.

Conclusion: The ESHRE/ESGE classification of female genital anomalies seems to fulfil the expectations and the needs of the experts of the field, but its clinical value needs to be proven in everyday practice.

Congenital malformations of the female genital tract are embryological maldevelopments of the müllerian or paramesonephric ducts, resulting in deviations from normal anatomy. It is a rather common and usually benign condition with a prevalence of $4-7 \%$ of newborn girls. In parallel publications in Gynecological Surgery and Human Reproduction, the Congenital Uterine Anomalies (CONUTA) common ESHRE-ESGE Working Group presents a new classification system for female reproductive tract anomalies, based on anatomy where embryological origin has been adopted as the secondary basic characteristic in the design of the main classes. This classification may also be useful for the paediatric endocrinologist caring for these patient groups.

\section{Disorders of sex development: an update}

Ostrer $\mathrm{H}$

Departments of Pathology, Genetics, and Pediatrics, Albert Einstein College of Medicine, Bronx, NY, USA

J Clin Endocrinol Metab 2014;99:1503-1509

Background: Disorders of sex development (DSDs) may arise from genetic defects in testis or ovarian determination, or from environmental cues or combinations of both. Current analytical technologies and improved understanding of major regulatory pathways have cast new insight into the genetic basis for these disorders.

Methods: A PubMed search was performed for the years 2011-13 using the terms 'disorder of sex development', 'gonadal dysgenesis', 'ovarian dysgenesis', 'array CGH', and 'whole exome sequencing'. Only articles from peer-reviewed journals were included. 
Results: Key themes that emerged included aberrant regulation of $S O X 9$ via the human testis-specific enhancer of Sox9 (hTES) promoter in 46,XY gonadal DSDs, the role of the MAPK pathway in normal and aberrant gonadal development, and the role of new technologies in identification of gonadal DSD.

Conclusion: Robust new technologies of array comparative genomic hybridization and genomic sequencing enabled many new sex-determining genes to be identified. These genes have been organized into ovarian- and testicular-determining pathways that can block each other's activities. Identification of a mutation in a sex-determining gene in an individual with DSD may warrant more extensive investigation for other phenotypic effects as well as genetic testing of other family members.

The incidence of DSD in the population is up to 1:1,000 according to this systematic literature review which investigated causes of DSDs. Primarily, gonadal disorders may be identified at different times of the life cycle. This includes newborns with ambiguous external genitalia, dysgenetic gonads, and internal genitalia that are discordant for the sex chromosome constitution. They may also be diagnosed later in life in individuals with delayed puberty, unanticipated virilization or gynaecomastia, infertility, or gonadal tumors. A correct diagnosis of an individual with DSD relies on clinical findings, hormonal analysis, gonadal histology, chromosome analysis, and genetic testing. Among the genes identified were SRY, SOX9, NR5A1, WT1, DAX1, WNT4, CBX2, DMRT1 and GATA4, and these have, with the new techniques available in recent years, been organized into ovarian- and testis-determining pathways. The author concludes that since the repertoire of genes can account for only a fraction of the genetic causes, more genes are likely to be identified. Moreover, epistatic interactions between variant-bearing genes will be identified that may account for phenotypic variations in the presentation of these disorders. This review is recommended to the paediatric endocrinologist who wants to remain updated on this expanding topic.

\section{New concerns}

\section{Population-based nationwide study of hypospadias in Sweden, 1973 to 2009: incidence and risk factors}

Nordenvall AS, Frisen L, Nordenstrom A, Lichtenstein P, Nordenskjold A

Department of Women's and Children's Health, Pediatric Surgery Unit and Center for Molecular Medicine,

Department of Clinical Neuroscience, Pediatric Endocrinology Unit, Astrid Lindgren Children's Hospital, Karolinska

University Hospital, and Department of Medical Epidemiology and Biostatistics, Karolinska Institutet, Stockholm,

Sweden

J Urol 2014;191:783-789

Background: The incidence of hypospadias in Sweden during a 40-year period was studied to determine if changes were associated with known risk factors.

Methods: Prospective data from nationwide healthcare and demographic registers collected for all males born in Sweden between 1973 and 2009 were analyzed. The incidence of hypospadias per 1,000 liveborn boys was calculated as number of cases divided by total number of births yearly. The association between hypospadias and risk factors was estimated using logistic regression, expressed as odds ratios. Results: The nationwide incidence of boys diagnosed with hypospadias was approximately 4.5 per 1,000 live-born boys until 1990, increasing to 8 per 1,000 boys during the following decade. Mild and severe phenotypes comprised the increase. Boys born small for gestational age (OR 4.34), as a twin (OR 1.8), as a result of in vitro fertilization (OR 1.15), or with parents from Asia (OR 1.45) or continental Europe (OR 1.41) were at increased risk for hypospadias. Multivariate analyses revealed that changes in risk factors did not explain the increased incidence. A systematic change in the classification of the diagnosis in registers could not be ruled out.

Conclusion: This nationwide study demonstrates an increased incidence of hypospadias diagnoses in Sweden from 1990 to 1999 that is not attributable to previously known risk factors. The increase includes mild and severe phenotypes, suggesting that shifts in the diagnostic criteria are not the underlying cause. 
Hypospadias is one of the most common congenital malformations in boys. There is evidence of an increased incidence of hypospadias without a relation to known risk factors. From this large nationwide registry-based study, the authors demonstrate that the incidence of hypospadias in Sweden increased during the late 20th century, reaching almost 8 per 1,000 male births. The risk of being diagnosed with hypospadias was about 50\% greater in 2006-2007 than in 1992-1993 or 1974-1975. The authors conclude that they have found an increased incidence of boys diagnosed with mild and severe hypospadias in Sweden that cannot be attributed to previously known risk factors either alone or in combination. Thus, the review raises concerns about possible unknown causes and whether there will be a continuing increase in the incidence of hypospadias. These results add to the ongoing discussion of possible environmental causes (such as endocrine disruptors), of the increased incidences of genital malformations in newborn boys, and later life reproductive disorders in males.

\section{New paradigms}

\section{Genetic loss or pharmacological blockade of testes-expressed taste genes causes male sterility}

Mosinger B, Redding KM, Parker MR, Yevshayeva V, Yee KK, Dyomina K, Li Y, Margolskee RF

Monell Chemical Senses Center, Philadelphia, PA, USA

Proc Natl Acad Sci USA 2013;110:12319-12324

Background: TAS1R taste receptors and their associated heterotrimeric $\mathrm{G}$ protein gustducin are involved in sugar and amino acid sensing in taste cells in the oral cavity and in the gastrointestinal tract. They are also strongly expressed in testis and sperm, but their functions in these tissues were previously unknown.

Methods: Using mouse models, it was shown that the genetic absence of both TAS1R3 and the gustducin $\alpha$-subunit GNAT3 lead to male-specific sterility. To gain further insight into this effect, the investigators generated a mouse model that expressed a humanized form of TAS1R3 that is known to be susceptible to inhibition by the antilipid medication clofibrate.

Results: Sperm formation in animals without functional TAS1R3 and GNAT3 is compromised, resulting in malformed and immotile sperm. Furthermore, clofibrate inhibition of humanized TAS1R3 in the genetic background of Tas1r3(-/-), Gnat3(-/-) doubly null mice led to inducible male sterility. These results indicate a crucial role for these extraoral 'taste' molecules in sperm development and maturation. It was previously reported that blocking of human TAS1R3, but not mouse TAS1R3, can be achieved by common medications or chemicals in the environment.

Conclusion: The authors hypothesized that even low levels of compounds that bind to taste receptors can reduce sperm count and negatively affect human male fertility, which common mouse toxicology assays would not reveal. Conversely, it was speculated that TAS1R3 and GNAT3 activators may help infertile men, particularly those who are affected by some of the mentioned inhibitors and/or are diagnosed with idiopathic infertility involving the signaling pathway of these receptors.

This interesting study reveals that cellular receptors used by oral taste cells are also expressed in the human testis and may have a role in testicular and sperm function. The findings also raise the possibility that molecules in the environment may bind and affect these receptors with possible consequences for male fertility. This spurs the ongoing debate on the role of endocrine disrupting chemicals in male infertility by adding novel mechanisms of their potential disruptive actions. Human but not mouse taste receptor TAS13 is inhibited by the antilipid drug clofibrate, but if and how this common drug in adult medicine affects male reproductive function is so far unknown. There are several potential implications of the present findings that need to be explored. One such path is the potential role of the taste receptors to serve as targets for male contraceptives. Another obvious question is whether taste sensations created by small molecules in the oral cavity may also be sensed directly by the testis and what biological implications such pathway might have. This study was highlighted in Biology of Reproduction [6]. 


\section{Two $Y$ genes can replace the entire $Y$ chromosome for assisted reproduction in the mouse}

Yamauchi Y, Riel JM, Stoytcheva Z, Ward MA

Institute for Biogenesis Research, John A. Burns School of Medicine, University of Hawaii, Honolulu, HI, USA

Science 2014;343:69-72

Background: The Y chromosome is crucial for male sex differentiation and reproduction. It was previously shown that, with the use of assisted reproduction, live offspring can be obtained from mice lacking the entire $\mathrm{Y}$ chromosome long arm, but exactly which genes are required has been unknown.

Methods and Results: In this study the investigators demonstrated that live mouse progeny can be generated by using germ cells from males with the Y chromosome contribution limited to only two genes, the testis determinant factor Sry and the spermatogonial proliferation factor Eif2s $3 y$.

Conclusion: Sry is believed to function primarily in sex determination during fetal life. Eif2s3y may be the only Y chromosome gene required to drive mouse spermatogenesis, allowing formation of haploid germ cells that are functional in assisted reproduction. These findings may be relevant for better understanding of mechanisms behind and treatment of human male infertility.

This study shows that only two genes on the $Y$ chromosome are necessary for a male mouse to produce offspring in an experimental setting requiring assisted reproduction. Sry is mandatory for testis determination creating host tissue for primordial germ cell immigration and spermatogenesis. Another gene, Eif2s3y, enabled spermatogonia to develop further through meiosis I and, in a few cells, also through meiosis II. Some of these haploid cells then developed into round spermatids which were injected into oocytes to produce offspring. The results may have implications for male infertility and its treatment. It is also apparent that the necessary male contribution to create an offspring is now reduced to only two genes. Combined with modern ART, this insight may have wide implications for the future. See also the paper by Bellott et al. cited below presenting a somewhat different view of the role of $Y$ chromosome located genes. This study was highlighted in Biology of Reproduction [7].

\section{Mammalian Y chromosomes retain widely expressed dosage-sensitive regulators}

Bellott DW, Hughes JF, Skaletsky H, Brown LG, Pyntikova T, Cho TJ, Koutseva N, Zaghlul S, Graves T, Rock S, Kremitzki C, Fulton RS, Dugan S, Ding Y, Morton D, Khan Z, Lewis L, Buhay C, Wang Q, Watt J, Holder M, Lee S, Nazareth L, Rozen S, Muzny DM, Warren WC, Gibbs RA, Wilson RK, Page DC

Whitehead Institute, Howard Hughes Medical Institute, and Department of Biology, Massachusetts Institute of Technology, Cambridge, MA, The Genome Institute, Washington University School of Medicine, St. Louis, MO, and Human Genome Sequencing Center, Baylor College of Medicine, Houston, TX, USA

Nature 2014;508:494-499

Background: The human X and Y chromosomes evolved from an ordinary pair of autosomes, but millions of years ago genetic decay ravaged the $\mathrm{Y}$ chromosome, and only three percent of its ancestral genes survived.

Methods: The authors reconstructed the evolution of the $\mathrm{Y}$ chromosome across eight mammalian species to identify biases in gene content and the selective pressures that preserved the surviving ancestral genes. Results: The results indicated that survival was nonrandom, and in 2 cases, was convergent across placental and marsupial mammals.

Conclusion: The authors concluded that the gene content of the $\mathrm{Y}$ chromosome became specialized through selection to maintain the ancestral dosage of homologous X-Y gene pairs that function as broadly expressed regulators of transcription, translation and protein stability. The investigators proposed that beyond its roles in testis determination and spermatogenesis, the $\mathrm{Y}$ chromosome is essential for male viability, and has unappreciated roles in Turner's syndrome and in phenotypic differences between the sexes in health and disease.

This study shows that the $Y$ chromosome in various mammals has been subject to abundant gene loss in early evolution. The remaining genes that are conserved across the $Y$ chromosome of surviving species of mammals have since then been remarkably stable. In humans, the level of variation 
between individuals is considerably lower on the $Y$ chromosome than on other chromosomes. The $Y$ chromosome is enriched with transcription-regulating genes. It is thus not only hosting genes mandatory for testis formation, male development and spermatogenesis. Instead this study suggests that the $\mathrm{Y}$ chromosome has an impact on gene regulation across the genome in males, potentially influencing biological functions throughout life and in many tissues. The interpretations and conclusions of this study are to some extent in conflict with the publication by Yamauchi and co-workers presented in this chapter. This study was commented in Nature [8].

\title{
Osteocalcin regulates murine and human fertility through a pancreas-bone-testis axis
}

\author{
Oury F, Ferron M, Huizhen W, Confavreux C, Xu L, Lacombe J, Srinivas P, Chamouni A, Lugani F, Lejeune H, \\ Kumar TR, Plotton I, Karsenty G \\ Department of Genetics and Development, Columbia University, New York, NY, USA \\ J Clin Invest 2013;123:2421-2433
}

Background: The osteoblast-derived hormone osteocalcin promotes testosterone biosynthesis in the mouse testis by binding to GPRC6A in Leydig cells. Interestingly, osteocalcin-deficient mice exhibit increased levels of luteinizing hormone $(\mathrm{LH})$, a pituitary hormone that regulates androgen synthesis by Leydig cells. This finding raises the question of whether LH regulates osteocalcin's reproductive effects. Further, there is growing evidence that osteocalcin levels are a reliable marker of insulin secretion and sensitivity and circulating levels of testosterone in humans, but the endocrine function of osteocalcin is unclear.

Methods and Results: Using mouse models, the authors found that osteocalcin and LH act in two parallel pathways and that osteocalcin-stimulated testosterone synthesis is positively regulated by bone resorption and insulin signaling in osteoblasts. To determine the importance of osteocalcin in humans, the investigators analyzed a cohort of patients with primary testicular failure and identified 2 individuals harboring the same heterozygous missense variant in one of the transmembrane domains of GPRC6A, which prevented the receptor from localizing to the cell membrane.

Conclusion: This study uncovers the existence of a second endocrine axis that is necessary for optimal male fertility in the mouse and suggests that osteocalcin modulates reproductive function in humans.

This well performed study showed that the osteoblast-secreted hormone osteocalcin is important for human male fertility. It has been found that osteocalcin promotes testosterone biosynthesis in murine testes via the G-protein-coupled receptor GPRC6A. The relationship between osteocalcin and luteinizing hormone (LH), the main regulator of testosterone production in Leydig cells, is not known. The authors showed that osteocalcin regulates male fertility in humans and in mice independently of LH. Infertile human male patients were selected for a phenotype similar to the one of mice lacking osteocalcin: sterility, low testosterone and high LH levels. Two oligospermic male patients out of 59 investigated harbored an identical transversion in a transmembrane domain of GPRC6A, which was absent in 942 control individuals with a normal quality and quantity of sperm. The mutation affected a highly conserved residue and acts as a dominant negative mutation, resulting in loss of function of the osteocalcin receptor. The authors propose that GPRC6A might be a new locus for primary testicular failure in humans, the cause of which is often unknown. Further insights into the clinical implications of this apparent bone-testis axis are highly warranted. This study was highlighted in Nature Reviews in Endocrinology [9]. 


\section{Maternal tract factors contribute to paternal seminal fluid impact on metabolic phenotype in offspring}

Bromfield JJ, Schjenken JE, Chin PY, Care AS, Jasper MJ, Robertson SA

The Robinson Institute and School of Paediatrics and Reproductive Health, University of Adelaide, Adelaide, SA,

Australia

Proc Natl Acad Sci USA 2014;111:2200-2205

Background: Paternal characteristics and exposures influence physiology and disease risks in offspring, but the mechanisms are mostly unknown. Seminal fluid, which affects female reproductive tract gene expression as well as sperm survival and integrity, provides one potential pathway.

Methods: The consequences for offspring of ablating the plasma fraction of seminal fluid by surgical excision of the seminal vesicle gland were evaluated in mice.

Results: Conception was substantially impaired and, when pregnancy did occur, placental hypertrophy was evident in late gestation. After birth, the growth trajectory and metabolic parameters of progeny were altered, more profoundly in males, who exhibited obesity, distorted metabolic hormones, reduced glucose tolerance, and hypertension. Altered offspring phenotype was partly attributable to sperm damage and partly to an effect of seminal fluid deficiency on the female tract, because increased adiposity was also evident in adult male progeny when normal two-cell embryos were transferred to females mated with seminal vesicle-excised males. Moreover, embryos developed in female tracts not exposed to seminal plasma were abnormal from the early cleavage stages, but culture in vitro partly alleviated this. Absence of seminal plasma was accompanied by downregulation of the embryotrophic factors Lif, Csf2, Il6, and Egf and upregulation of the apoptosis-inducing factor Trail in the oviduct.

Conclusion: Thus, paternal seminal fluid composition affects the growth and health of male offspring and reveals that its impact on the periconception environment involves not only sperm protection but also indirect effects on the preimplantation embryo via oviduct expression of embryotrophic cytokines and growth factors.

Events at conception shape the future growth and health of the offspring, and may have impact on life expectancy and disease susceptibility. The environment and experiences of both parents contribute to programming offspring phenotype via epigenetic modifications taking place before embryo implantation. How the father transmits this information remains elusive. Normal females were mated with mice lacking seminal fluid. This resulted in many of the embryos failing to develop past the eight-cell stage, and the placentas of the offspring were unusually heavy. Moreover, the adult offspring had high rates of obesity and hypertension, aberrant levels of metabolic hormones, and reduced glucose tolerance. Thus, some factors in the seminal fluid convey epigenetic information to the embryo during the early cleavage stages, when the embryonic DNA is undergoing epigenetic reprogramming. These findings are consistent with emerging studies showing that stressful events, chemical exposures, and dietary conditions of a male can affect his offspring. The study was highlighted in Biology of Reproduction [10].

\section{Seminal vesicle protein SVS2 is required for sperm survival in the uterus}

Kawano N, Araki N, Yoshida K, Hibino T, Ohnami N, Makino M, Kanai S, Hasuwa H, Yoshida M, Miyado K, Umezawa A

Department of Reproductive Biology, National Center for Child Health and Development, Tokyo, Japan Proc Natl Acad Sci USA 2014;111:4145-4150

Background: In mammals, sperm migrate through the female reproductive tract to reach the egg but the understanding of this journey is highly limited. Male mice lacking seminal vesicle secretion 2 (SVS2) protein, a major component of seminal vesicle secretions, display prominently reduced fertility. However, their epididymal sperms are able to fertilize eggs normally in vitro, suggesting that SVS2 is only essential for in vivo fertilization. To shed light on this process, functions of seminal vesicle secretion 2 (SVS2) were defined. 
Methods: SVS2(-/-) males were generated and used for mating experiments, both in vivo and in vitro, in comparison to wild type mice.

Results: SVS2(-/-) male mice produced sperm but were severely subfertile, and formation of a copulatory plug to cover the female genital opening did not occur. Surprisingly, even when artificial insemination was performed with silicon as a substitute for the plug, sperm fertility in the absence of SVS2 remained severely reduced because the sperm were already dead in the uterus.

Conclusion: Thus, our results provide evidence that the uterus induces sperm cell death and that SVS2 protects sperm from uterine attack.

Infertility in mice lacking the SVS2 gene is not only caused by failed copulatory plug formation, but also by the disruption of ejaculated sperms in the uterus by uterus-derived cytotoxic factors. SVS2 seems to protect the sperm against uterus-derived cytotoxic factors by coating the sperm surface to prevent a uterine attack. Thus, these results provide evidence that mammalian males have developed a protective strategy against female attack at the gamete level. These observations have implications for male infertility and may be developed to a target of male contraceptives. This study was also highlighted in Biology of Reproduction [11].

\title{
Placental programming of anxiety in adulthood revealed by lgf2-null models
}

\author{
Mikaelsson MA, Constancia M, Dent CL, Wilkinson LS, Humby T \\ Behavioral Genetics Group, School of Psychology, MRC Centre for Neuropsychiatric Genetics and Genomics, \\ Neuroscience and Mental Health Research Institute, Cardiff University, Cardiff, UK \\ Nat Commun 2013:4:2311
}

Background: Imprinted, maternally silenced insulin-like growth factor- 2 is expressed in both the fetus and placenta and has roles in fetal and placental development in animal models.

Methods: Here the authors compared mice engineered to be null for the placenta-specific P0 transcript (insulin-like growth factor-2-P0 KO) to mice with disruptions of all four insulin-like growth factor-2 transcripts, and therefore null for insulin-like growth factor-2 in both placenta and fetus (insulin-like growth factor-2-total $\mathrm{KO}$ ).

Results: Both models lead to intrauterine growth restriction but dissociate between a situation where there is an imbalance between fetal demand and placental supply of nutrients (the insulin-like growth factor-2-P0 KO) and one where demand and supply is more balanced (the insulin-like growth factor2-total KO). Increased reactivity to anxiety-provoking stimuli is manifested later in life only in those animals where there is a mismatch between placental supply and fetal demand for nutrients during gestation.

Conclusion: Our findings further distinguish placental dysfunction from intrauterine growth restriction and reveal a role for the placenta in long-term programming of emotional behaviour.

Insulin-like growth factor 2 (IGF-2) is abundantly expressed in the fetus and binds the type 1 IGF receptor, but its precise physiological role in fetal and later life still remains to be elucidated. Imprinted, maternally silenced Igf2 is known to be expressed in both the fetus and placenta and has been shown to have a role in fetal and placental development in animal models. This study showed that an imbalance in IGF2 expression between the placenta and fetus influences the development of emotional behavior. The biological mechanisms behind this finding that events occurring before birth can influence emotional behavior later in life are obscure. These observations extend the prenatal origin of adult disease hypothesis to include also psychiatric disorders. Whether the observed effect is affecting the nervous system directly or relayed via intermediate cues remains to be elucidated. Nevertheless, the clinical implications of these findings, if proven to be correct by further studies, are wide. This paper was highlighted in Nature Reviews Endocrinology [12]. 


\section{Are young adult women with polycystic ovary syndrome slipping through the healthcare cracks?}

Dokras A, Witchel SF

Obstetrics and Gynecology, University of Pennsylvania, Philadelphia, PA, USA

J Clin Endocrinol Metab 2014,99:1583-1585

Background: Polycystic ovary syndrome (PCOS) is a common endocrine disorder often diagnosed in adolescence or early adulthood. In adolescence, the many similarities between normal features of puberty and symptoms of PCOS make it challenging to confirm the diagnosis. Even among adult women, the changing definitions of PCOS may lead to inaccurate diagnoses. Women may present with a variety of symptoms to different healthcare providers, and may be treated only for the presenting symptoms without evaluation of the syndrome and its associated morbidities. Timely evaluations, accurate diagnosis, appropriate interventions, and multidisciplinary healthcare teams can be valuable because women with PCOS have an increased risk for obesity, impaired glucose tolerance, diabetes, dyslipidemia, metabolic syndrome, infertility, endometrial cancer and anxiety and mood disorders.

Results: Appropriate transition of care for the adolescent from pediatric to adult healthcare providers should include education of the patient and her parents regarding the chronic nature of the syndrome and need for continued follow-up. Girls with symptoms suggestive of PCOS who fail to fulfill diagnostic criteria should undergo prolonged observation.

Conclusion: Early identification of PCOS at different entry points in the healthcare system will require physician education and improved access.

The incidence of polycystic ovary syndrome (PCOS) is increasing in many countries also involving the pediatric population. This text on PCOS in young women highlights the importance of early identification in affected girls, since early intervention with lifestyle changes could improve the long-term outcome. In this aspect it is also important with an appropriate transition of care when the girl is leaving the pediatric age group and links to the adult healthcare providers.

\section{Reproductive endocrinology: new guidelines for the diagnosis and treatment of PCOS}

Orio F, Palomba S

Department of Sports Science and Wellness, 'Parthenope' University Naples, Naples and Unit of Obstetrics and Gynaecology, Arcispedale 'Santa Maria Nuova', IRCCS, University of Modena and Reggio Emilia, Reggio Emilia, Italy Nat Rev Endocrinol 2014;10:130-132

Background: Polycystic ovary syndrome (PCOS) is a common and very heterogeneous condition characterized by clinical and/or biochemical androgen excess, ovulatory dysfunction and polycystic ovaries (PCO). The diagnosis of PCOS in adolescent women is considered elusive as the specific features of PCOS and their combination as diagnostic criteria have not been validated. This topic is very important given that early diagnosis and early intervention (e.g. lifestyle modifications) in adolescents could modify the natural history of PCOS.

Methods: An Endocrine Society-appointed task force has developed an evidence-based clinical practice guideline for the diagnosis and treatment of PCOS.

Results and Conclusion: The guidelines provide suggestions for the management of patients with PCOS and highlight many areas of uncertainty requiring further scientific efforts.

Although this publication mainly focuses on adults there are also implications for the adolescent age group. The new US Endocrine Society guidelines for diagnosis and treatment [13] support previous meta-analyses and guidelines in rejecting the use of metformin as part of the infertility treatment in PCOS and suggest the use of metformin only in women with glucose intolerance. Recent clinical evidence-based guidelines highlight that PCOS is a threat to women's general health. PCOS should not be considered only a fertility and cosmetic disorder, but rather a condition with long-term health risks. The treatment of PCOS should be tailored to the patients' and the physicians' therapeutic goals, since no efficient single therapy is currently available. Lifestyle changes including diet and physical activity constitute a main focus of the therapeutic interven- 
tions. Success is more likely if therapeutic activities are funded and implemented at an early age. Therefore, these guidelines are also important for the pediatric endocrinologist caring for children with PCOS.

\title{
Food for thought
}

\section{Why do female Callosobruchus maculatus kick their mates?}

van Lieshout E, McNamara KB, Simmons LW

Centre for Evolutionary Biology, School of Animal Biology (M092), University of Western Australia, Crawley, WA, Australia

PLoS One 2014;9:e95747

Background: Sexual conflict is recognized as an important driver of sexual trait evolution. However, due to their variable outcomes and effects on other fitness components, the detection of sexual conflicts on individual traits can be complicated. This difficulty is exemplified in the beetle Callosobruchus macula$t u s$, where longer matings increase the size of nutritious ejaculates but simultaneously reduce female future receptivity. While previous studies show that females gain direct benefits from extended mating duration, females show conspicuous copulatory kicking behaviour, apparently to dislodge mating males prematurely.

Methods: The authors explored the potential for sexual conflict by comparing several fitness components and remating propensity in pairs of full sibling females where each female mated with a male from an unrelated pair of full sibling males. For one female, matings were terminated at the onset of kicking, whereas the other's matings remained uninterrupted.

Results: While fecundity (number of eggs) was similar between treatments, uninterrupted matings enhanced adult offspring numbers and fractionally also increased longevity. However, females whose matings were interrupted at the onset of kicking exhibited an increased propensity to remate.

Conclusion: Since polyandry can benefit female fitness in this species, the investigators argued that kicking, rather than being maladaptive, may indicate that females prefer remating over increased ejaculate size. It may thus be difficult to assess the presence of sexual conflict over contested traits such as mating duration in models where females face a trade-off between direct benefits gained from one mating and indirect benefits from additional matings.

This is a study on sexual behavior in a lower species, the Callosobruchus beetle. It deals with sexual conflict, which in this context is defined as the conflict between the evolutionary interests of males and females, constituting an important driver of adaptation. It was shown that some female fitness components clearly benefit from longer copulations. By enforcing mating durations to be closer to the female optima, the authors found indications that the direct fitness benefits from mating duration may trade off with genetic benefits gained through additional polyandrous mating. The translation of these results to mammals including humans may be far-fetched but such exercise may still be a stimulating endeavor.

\section{Changes in sleep time and sleep quality across the ovulatory cycle as a function of fertility and partner attractiveness}

\author{
Gentle BN, Pillsworth EG, Goetz AT
}

Department of Psychology and Social Behavior, University of California, Irvine, and Departments of Anthropology and Psychology, California State University, Fullerton, CA, USA

PLoS One 2014;9:e92796

Background: Research suggests that near ovulation women tend to consume fewer calories and engage in more physical activity; they are judged to be more attractive, express greater preferences for masculine and symmetrical men, and experience increases in sexual desire for men other than their primary partners. Some of these cycle phase shifts are moderated by partner attractiveness and interpreted as strategic responses to women's current reproductive context. 
Methods: The present study investigated changes in sleep across the ovulatory cycle, based on the hypothesis that changes in sleep may reflect ancestral strategic shifts of time and energy toward reproductive activities. Participants completed a 32-day daily diary in which they recorded their sleep time and quality for each day, yielding over 1,000 observations of sleep time and quality. Women in relationships rated the physical and sexual attractiveness of their current partner using two items in the survey, 'How physically attractive would others rate your partner?' and 'How sexually attractive would others rate your partner?' Each variable was rated from 1 ('not physically [sexually] attractive') to 9 ('very physically [sexually] attractive'). These variables were summed to create a single measure of partner attractiveness $(\mathrm{a}=0.924)$, with a possible score of $2-18$ (mean 14.05, SD 2.17, range 10-18).

Results: The results indicated that when probability of conception was high, women partnered with less attractive men slept more, while women with more attractive partners slept less.

Conclusion: The ovulatory shift hypothesis proposes that women will experience systematic shifts in desires and mating-related behaviors when most likely to conceive in order to increase the likelihood of reproducing with genetically fit partners and decrease the likelihood of reproducing with genetically less fit partners. Many studies have produced evidence that women prefer more masculine and more symmetrical men as sexual partners at high fertility relative to low. This hypothesis is supported by the present findings.

This is an interesting study on sexual behavior in human females in relation to the menstrual cycle and probability to conceive. The main finding was that women with more sexually attractive partners (according to self-reported criteria) slept less when the probability of conception was higher, while women with less attractive partners slept more. The decreased sleep at high fertility periods may reflect a strategic shift to reproductive effort when conception is most likely. The authors also suggest that women with less attractive partners are strategically avoiding conception. How these strategies affect everyday health and wellbeing of women remains to be studied. It would also be of interest to do a parallel study to investigate how the present findings in females were mirrored in their male partners. The endocrine and neural signaling pathways involved in the investigated processes are yet to be defined. They could easily be imagined as interesting targets of intervention.

\section{References}

1. Reproductive endocrinology: Aromatase (TTTA)13 repeat increases risk of central precocious puberty. Nat Rev Endocrinol 2014;10:251.

2. Kugelberg E: Reproductive endocrinology: ESR1 mutation causes estrogen resistance and puberty delay in women. Nat Rev Endocrinol 2013;9:565.

3. Lubahn DB, Moyer JS, Golding TS, Couse JF, Korach KS, Smithies O: Alteration of reproductive function but not prenatal sexual development after insertional disruption of the mouse estrogen receptor gene. Proc Natl Acad Sci USA 1993;90:11162-11166

4. Smith EP, Boyd J, Frank GR, Takahashi H, Cohen RM, Specker B, et al: Estrogen resistance caused by a mutation in the estrogen-receptor gene in a man. N Engl J Med 1994;331:1056-1061.

5. Schubert C: World of reproductive biology: putting together a primordial germ cell. Biol Reprod 2013, July 17, 2013.

6. Schubert C: World of reproductive biology: taste receptors may spell trouble for testes. Biol Reprod 2013, July 10, 2013.

7. Schubert C: World of reproductive biology: maleness minimized to two genes. Biol Reprod 2013, Nov 27, 2013.

8. Clark AG: Genetics: the vital Y chromosome. Nature 2014;508:463-465.

9. Kugelberg E: Reproductive endocrinology: osteocalcin and male fertility. Nat Rev Endocrinol 2013;9:441.

10. Schubert C: World of reproductive biology: a little seminal fluid goes a long way. Biol Reprod 2014, Jan $29,2014$.

11. Schubert C: World of reproductive biology: molecular protector shields sperm from death. Biol Reprod 2014, Mar 26, 2014.

12. Reproductive endocrinology: Insulin-like growth factor 2 and long-term placental programming of anxiety. Nat Rev Endocrinol 2013;9:566-570.

13. Legro RS, Arslanian SA, Ehrmann DA, Hoeger KM, Murad MH, Pasquali R, et al: Diagnosis and treatment of polycystic ovary syndrome: an Endocrine Society clinical practice guideline. J Clin Endocrinol Metab 2013;98:4565-4592. 